\title{
Effect of the chitin synthesis inhibitor triflumuron on the development, viability and reproduction of Aedes aegypti
}

\author{
Thiago Affonso Belinato ${ }^{1,2}$, Ademir Jesus Martins ${ }^{1,2}$, José Bento Pereira Lima ${ }^{1,2}$, \\ Tamara Nunes de Lima-Camara ${ }^{3}$, Alexandre Afrânio Peixoto ${ }^{3}$, Denise Valle $\mathrm{e}^{1,2 /+}$
}

${ }^{1}$ Laboratório de Fisiologia e Controle de Artrópodes Vetores ${ }^{3}$ Laboratório de Biologia Molecular de Insetos Instituto Oswaldo Cruz-Fiocruz, Av. Brasil 4365, 21045-900 Rio de Janeiro, RJ, Brasil 'Laboratório de Entomologia, Instituto de Biologia do Exército, Rio de Janeiro, RJ, Brasil

The control of Aedes aegypti is impaired due to the development of resistance to chemical insecticides. Insect Growth Regulators (IGR) exhibit distinct mechanisms of action and are considered potential vector control alternatives. Studies regarding the effects of sublethal IGR doses on the viability of resulting adults will contribute to evaluating their impact in the field. We analyzed several aspects of Ae. aegypti adults surviving exposure to a partially lethal dose of triflumuron, a chitin synthesis inhibitor. A highly significant difference in the proportion of males and females was noted in the triflumuron-exposed group (65.0\% males) compared to the controls (50.2\% males). Triflumuron affected adult longevity, particularly for females; after 16 days, only $29.2 \%$ of males and $13.8 \%$ of females were alive, in contrast with $94 \%$ survival of the control mosquitoes. The locomotor activity was reduced and the blood-feeding ability of the treated females was also affected $(90.4 \%$ and $48.4 \%$ of the control and triflumuronexposed females, respectively, successfully ingested blood). Triflumuron-surviving females ingested roughly $30 \%$ less blood and laid 25\% fewer eggs than the control females. The treated males and females exhibited a diminished ability to copulate, resulting in less viable eggs.

Key words: triflumuron - Aedes aegypti - sublethal effects - chitin synthesis inhibitor

The mosquito Aedes aegypti, widely distributed in tropical and subtropical regions, is highly adapted to the urban environment. It is frequently found inside or near houses and it plays an important role in the transmission of arboviruses such as dengue and urban yellow fever (Consoli \& Lourenço-de-Oliveira 1994, Gubler 2002).

Currently, Ae. aegypti control is mainly performed with chemical larvicides that target the insect's central nervous system. However, the long history of insecticide use has led to the development of resistant populations all over the world. In this sense, the study of novel tools to control Ae. aegypti and other insects of medical importance is a major area of interest (Zaim \& Guillet 2002).

An alternative vector control approach is the use of compounds with distinct mechanisms of action and target sites, like the Insect Growth Regulators (IGR). These include the juvenile hormone analogues, the ecdysone agonists and the chitin synthesis inhibitors (CSIs) (Graf 1993, Tunaz \& Uygun 2004).

CSIs are benzoylphenylurea compounds discovered in the 1970s (Mian \& Mula 1982) that interfere with insect development, disturbing the moult and resulting in deformations in the cuticle (Reynolds 1987). These alter-

Financial support: CNPq, FAPERJ, FIOCRUZ, SVS-MS

+ Corresponding author:dvalle@ioc.fiocruz.br

Received 5 September 2008

Accepted 11 December 2008 ations occur due to CSI interference in chitin synthesis, diminishing the amount of this polymer in the insect cuticle (Ishaaya \& Casida 1974, Post et al. 1974). Additionally, adults deriving from CSI-exposed larvae can exhibit a series of physiological constraints that ultimately lead to diminished physical and reproductive fitness (Mondal $\&$ Parween 2000). These CSI effects vary according to the species, the developmental stage at the time of application, the kind of compound and the administered dose (Vasuki \& Rajavel 1992, Wilson \& Cryan 1997, Mulla et al. 2003). Although several reports in the literature explore the direct effects of CSIs, such as mortality or adult emergence inhibition (Rehini \& Soltani 1999, Su et al. 2003, Batra et al. 2005), the consequences of IGR use on the surviving adults and their implications in vector fitness are less considered, especially among Culicidae (Vasuki 1992, 1999, Vasuki \& Rajavel 1992).

Ae. aegypti is a domestic mosquito whose breeding sites (mostly water storage units for human use) are subjected to frequent volume variations, resulting in a decrease of the insecticide residual effect. In this sense, detailed studies of the effects of sublethal CSI doses on the viability of resulting adults will contribute to evaluating their impact in the field.

We previously confirmed the effectiveness of triflumuron against field Ae. aegypti populations, including one strain resistant to the organophosphate temephos (Martins et al. 2008). In the present study, we evaluated the effects of exposing Ae. aegypti larvae to a partially lethal dose of the CSI triflumuron on the resulting adults. Several parameters were investigated, including the proportion of surviving males and females, adult longevity and activity, blood feeding, mating and viability of the eggs. 


\section{MATERIALS AND METHODS}

Mosquitoes - Mosquitoes from the Rockefeller strain, an insecticide-susceptible reference lineage, were used in all assays. Rearing conditions were performed at $25 \pm 1^{\circ} \mathrm{C}$ and $80 \%$ rh following standard procedures (Braga et al. 2005).

Bioassays - Starycide SC $0.48^{\circledR}$ (Bayer) was employed to prepare a stock solution of triflumuron at $48 \mu \mathrm{g} / \mathrm{mL}$ in DMSO (dimethyl sulfoxide) and aliquots were stored at $-80^{\circ} \mathrm{C}$. Working solutions were prepared in dechlorinated water. Transparent plastic cups with groups of 10 L3 larvae were exposed to $150 \mathrm{~mL}$ of $0.7-0.9 \mu \mathrm{g} / \mathrm{L}$ triflumuron solution, concentrations close to the $\mathrm{EI}_{50}$ dose (Martins et al. 2008). One milliliter of a $2.5 \%$ (w/v) solution of ground cat food (Friskies ${ }^{\circledR}$, Purina, Camaquã/RS) was supplied once. Control larvae were exposed to the same DMSO volume used in the triflumuron samples $(2 \mu \mathrm{L})$. Dead specimens and resulting adults were collected daily. Each bioassay was composed of eight experimental and three control cups, with each cup containing 10 larvae.

Male/female rate - Newly emerged adults surviving triflumuron exposure were removed daily and the numbers of males and females from both the experimental and control conditions were scored. The resulting adults were used for longevity and reproduction assays.

Longevity - Pools of 15 females and 15 males, derived from triflumuron-exposed or control larvae, were placed in small carton cages $(8.5 \mathrm{~cm}$ diameter x $8.5 \mathrm{~cm}$ high) and fed continuously with a $10 \%$ sucrose solution. Mortality was registered daily over 16 days. Each assay was composed of three experimental and three control cages.

Activity - The activity pattern of triflumuron-surviving and control Ae. aegypti females was evaluated with a Locomotor Activity Monitor (TriKinetics) (Gentile et al. 2006). Two-day old mosquitoes were individually placed in glass tubes with a cotton plug soaked in $10 \%$ sucrose solution and the tubes were placed in the Monitor inside a Precision Scientific Incubator Mod. 818 under constant temperature $\left(25^{\circ} \mathrm{C}\right)$ and a $12 \mathrm{~h}$ light: $12 \mathrm{~h}$ dark photoperiod (LD 12:12). The locomotor activity was individually registered every time a mosquito crossed the middle of the tube, interrupting an incident infrared light. For every mosquito, 48 data points (representing the total locomotor activity of $30 \mathrm{~min}$ intervals) were obtained for every day of monitoring. Only data from mosquitoes that were alive for the first three days of monitoring were considered for the analysis, which was performed through calculation of the Williams mean of their activity. This assay was performed twice.

Blood feeding - Three-day old adult females were exposed to an anesthetized guinea pig for $30 \mathrm{~min}$. The number of females that successfully engorged was registered for both the experimental and control groups. In order to quantify the amount of ingested blood, distinct groups of 10 experimental or control mosquitoes were weighed in an analytic balance (APX-200, Denver Instrument) before or after the blood meal. The amount of ingested blood was calculated by subtraction of these mean values.

Egglaying - Egglaying was induced in individual females three days after the blood meal, according to the procedure adapted from Valencia et al. (1996), using inverted Petri dishes, at $26^{\circ} \mathrm{C}$, with a wet filter paper at the lid. The numbers of successful egglaying females and of eggs per female were investigated, as well as the viability of the eggs.

Rate of inseminated females - Individual couples formed from (1) triflumuron-surviving females and control males or, reciprocally, (2) triflumuron survivingmales and control females, (3) triflumuron-surviving males and females and (4) control males and females were placed in $50 \mathrm{~mL}$ transparent plastic tubes. Males and females were obtained from pupae reared individually, in order to assure that virgin females would be used. After three days, female spermathecae were dissected and sperm presence was observed with an optic microscope (Nikon Biophot, 200X).

Statistical analysis - Unless stated, each parameter was evaluated in replicates and repeated three times. Triflumuron-surviving and control groups were compared by $t$ test or $\chi^{2}$ analysis as indicated, using GraphPad Prism version 4.00 for Windows (GraphPad Software, San Diego California USA, www.graphpad.com).

\section{RESULTS}

Several bioassays were performed to accomplish the analysis shown here. From a total of 12,000 experimental specimens exposed to triflumuron, $43.6 \%$ adult emergence was obtained. A delay in larvae development was not noted in the experimental group, when compared to controls; in both cases, adult emergence took 5-7 days after the onset of the assays. On the other hand, several alterations in aspects related to viability and reproduction were noted in the experimental group, as follows.

Viability aspects - In the control group, equivalent rates of males $(50.2 \pm 4.1 \%)$ and females were obtained, while males accounted for $65.0 \% \pm 7.6 \%$ of the triflumuron-surviving adults, a highly significant difference between both sexes $\left(\chi_{0.05,1}^{2}=75.82 ; \mathrm{p}<0.0001\right)$. This represents male/female proportions of 1.01 and 1.86 in the control and experimental groups, respectively.

Fig. 1 shows the daily mortality of males and females from the control and experimental groups up to 16 days after adult emergence. In opposition to the control group, with total mortality below $6 \%$ for both males and females during this period, triflumuron survivors exhibited a reduced longevity. Moreover, females were more affected than males; after 16 days, the rate of surviving males $(29.2 \%)$ was twice that of females $(13.8 \%)$.

Ae. aegypti adults show diurnal habits, with peaks of activity at the beginning and at the end of the photophase. Their pattern of activity was not altered by triflumuron exposure. However, the intensity of activity was significantly reduced on photophase $\left(t_{0.05(2), 139} ; \mathrm{p}<0.05\right)$, as exemplified in Fig. 2. 
The ability to ingest blood and the feeding rate were significantly altered in CSI-surviving females; while $90.4 \%(85 / 94)$ of control females accepted the blood meal, this rate decreased to $48.4 \%$ (77/159) for triflumuron-exposed females $\left(\chi_{0.05,1}^{2}=9.44 ; \mathrm{p}<0.005\right)$. Moreover, the control and triflumuron-surviving females ingested, respectively, $2.57 \pm 0.58$ and $1.79 \pm 0.28$ times their weight in blood, which also shows a significant difference $\left(t_{0.05(1), 17 ;} \mathrm{p}<0.05\right)$. In spite of this, the weights of non-blood-fed control $(7.37 \pm 0.66 \mu \mathrm{g})$ and triflumuronsurviving $(7.71 \pm 1.02 \mu \mathrm{g})$ females after adult emergence were equivalent.

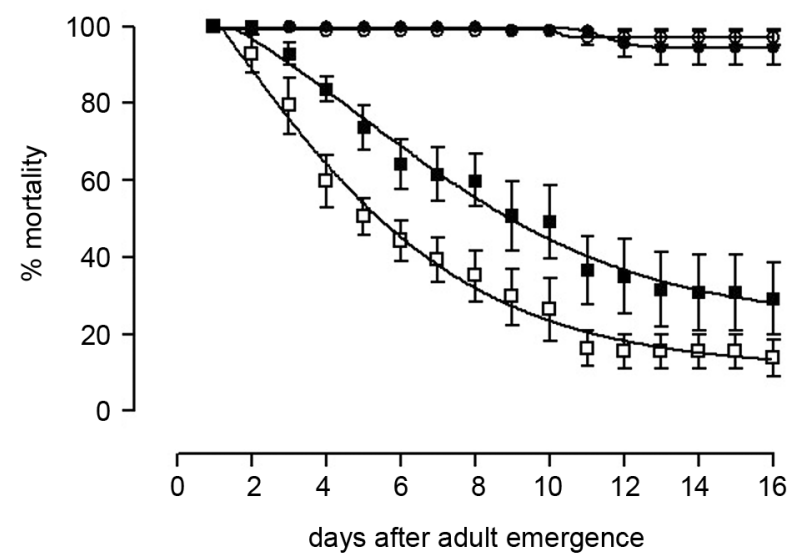

Fig. 1: daily mortality of control (circles) and triflumuron-surviving (squares) Aedes aegypti adults. Opened and closed symbols refer to females and males, respectively.

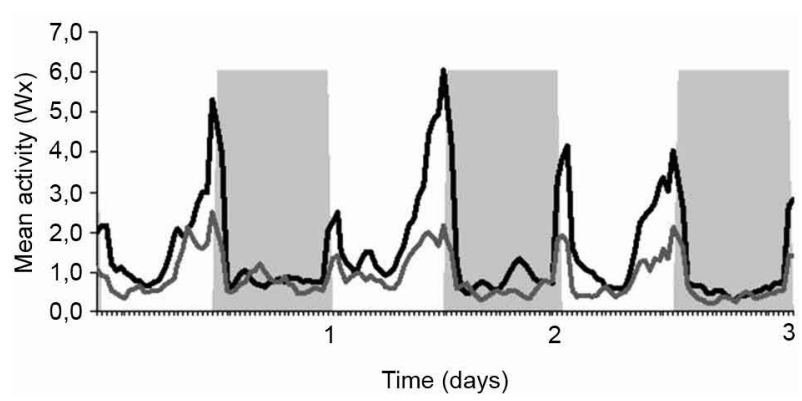

Fig. 2: Aedes aegypti females activity rhythm during three days under a 12:12 light/dark (gray bars) regimen. Black and gray lines represent control and triflumuron-surviving females, respectively.
Reproduction aspects - Table I summarises the oviposition data of the control and triflumuron-exposed females. Approximately $10 \%$ of the experimental blood-fed females died up to $24 \mathrm{~h}$ after blood feeding and an even higher mortality ( $35 \%$ ) was observed after egglaying. The number of eggs per female was also significantly different in both groups $\left(t_{0.05(2) .222} ; \mathrm{p}<0.0001\right)$.

Among the triflumuron-surviving females that accomplished oviposition, $14.4 \%$ presented eggs that broke when immersed in water (Fig. 3). Spermathecae dissection revealed that these females had not been inseminated. On the other hand, no significant difference was noted in the viability of eggs derived from triflumuron-treated females that had effectively been inseminated $(96.3 \% \pm 1.2 \%)$ compared to those from control females $(97.5 \% \pm 1.0 \%)$.

In order to verify if triflumuron preferentially interferes with the reproductive ability of one sex, individual matings with one control partner and one triflumuron survivor were performed. Although 75 couples were formed for each condition, at the end of three days one or both partners from several couples had died (Table II). When the surviving couples were considered, a reduction in the rate of inseminated females was noted in all groups bearing at least one triflumuron-treated mate (Table II). There was no significant difference $\left(\chi^{2}, \mathrm{p}>0.05\right)$ among the three groups of couples containing at least one treated individual, but significant differences were noted when any of them was compared to the group of couples formed by non-treated individuals $\left(\chi^{2}, \mathrm{p}<0.05\right)$.

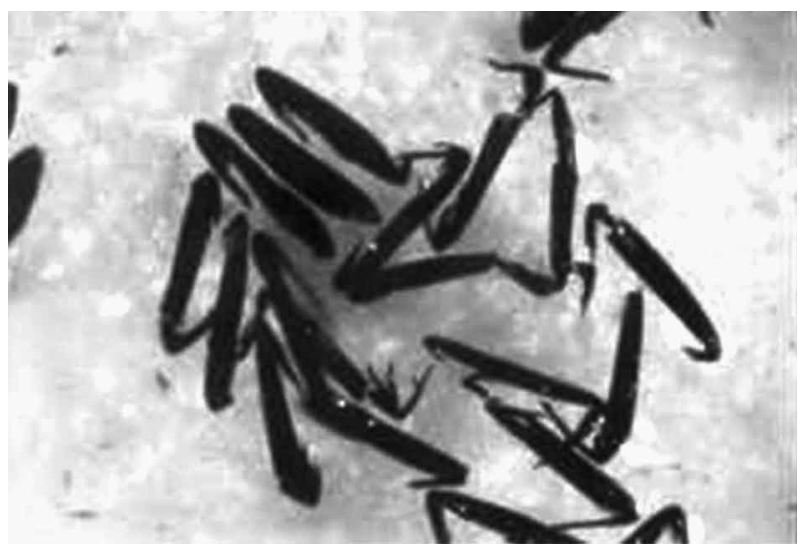

Fig. 3: eggs laid by Aedes aegypti triflumuron-surviving females. Broken eggs derive from unfertilized specimens.

TABLE I

Oviposition of Aedes aegypti triflumuron-surviving adult females

\begin{tabular}{lccccc}
\hline Group & $\mathrm{n}$ & $\begin{array}{c}\text { Mortality after } \\
\text { blood meal }\end{array}$ & $\begin{array}{c}\text { Females } \\
\text { without eggs }\end{array}$ & $\begin{array}{c}\text { Mortality after } \\
\text { egglaying }\end{array}$ & $\begin{array}{c}\text { Mean } \\
\text { eggs/females }\end{array}$ \\
\hline Control & 100 & 1 & 6 & 1 & $112.9 \pm 41.1$ \\
Triflumuron & 139 & 14 & 14 & 44 & $86.4 \pm 44.0^{a}$ \\
\hline
\end{tabular}

All data refer to the total number of adult females tested (n), which is the sum of the three assays performed. Exception is the last column, where only egglaying females were considered. $a: \mathrm{p}<0.0001$ compared to control. 


\section{TABLE II}

Rate of inseminated females resulting from individual matings between control and triflumuron-surviving Aedes aegypti adults

\begin{tabular}{|c|c|c|}
\hline Group & $\begin{array}{c}\text { Surviving couples }{ }^{a} \\
\text { n (\%) }\end{array}$ & $\begin{array}{c}\text { Inseminated females } \\
\mathrm{n}(\%)\end{array}$ \\
\hline$\delta$ cont $\mathrm{x}$ + cont & $75(100)$ & $74(99)^{b}$ \\
\hline$\partial$ cont $x \rightarrow$ trif & $45(60)$ & $15(33)^{c}$ \\
\hline$\delta$ trif $x+$ cont & $66(88)$ & $22(33)^{c}$ \\
\hline$\delta$ trif $x+$ trif & $44(59)$ & $8(18)^{c}$ \\
\hline
\end{tabular}

$a$ : 75 individual couples were made for each experimental condition; $b, c$ : values followed by the same letter do not differ significantly; cont: control; trif: adult surviving from triflumuron exposure, performed in the larval stage.

\section{DISCUSSION}

Our data indicate that the exposure of Ae. aegypti larvae to sublethal doses of the CSI triflumuron results in adult mosquitoes affected in several aspects of their viability and reproductive ability.

Disequilibrium in the proportion of males and females was observed after treatment with triflumuron at the $\mathrm{EI}_{50}$. The smaller proportion of females was attributed to their slower developmental kinetics when compared to males (Clements 1992). As a consequence, females would be exposed to the CSI for a longer period of time in the larval stage. The exposure of the Coleoptera Tribolium confusum to triflumuron also resulted in a greater proportion of males (3:1) (El-Sayed et al. 1984).

Adult longevity was highly reduced in the triflumuron-treated specimens. Females were more severely affected than males, probably due to their longer period of contact with the CSI during the larval stage, as mentioned above. In contrast, treatment with the CSI hexaflumuron resulted in a drastic but equivalent reduction in the longevity of males and females of Culex quinquefasciatus, Anopheles stephensi and Ae. aegypti (Vasuki 1992), suggesting subtle variations among different CSIs. Triflumuron diminished the longevity of the triatomine Rhodnius prolixus when administered by contact, blood ingestion or injection (Mello et al. 2008). The coleoptera Tenebrio molitor exposed to the CSI diflubenzuron also exhibited reduced longevity (Soltani et al. 1983).

Triflumuron also reduced Ae. aegypti activity. Many triflumuron-surviving adults had structural abnormalities, such as deformed wings or easily broken legs, which could account for this reduced activity (Fig. 4). There are reports of structural defects produced by treatment with CSI in several insect orders (Demark \& Bennet 1989, Wilson \& Cryan 1997, Da-Silva et al. 2004). Vasuki and Rajavel (1992) observed that treatment of the mosquitoes Cx. quinquefasciatus, Ae. aegypti and An. stephensi with a CSI resulted in several visible abnormalities that could hamper locomotion and that were probably related to the reduced longevity of adults. Accordingly, Amir and Peveling (2004) verified that triflumuron-exposed Apis mellifera specimens exhibited a lower flight activity.

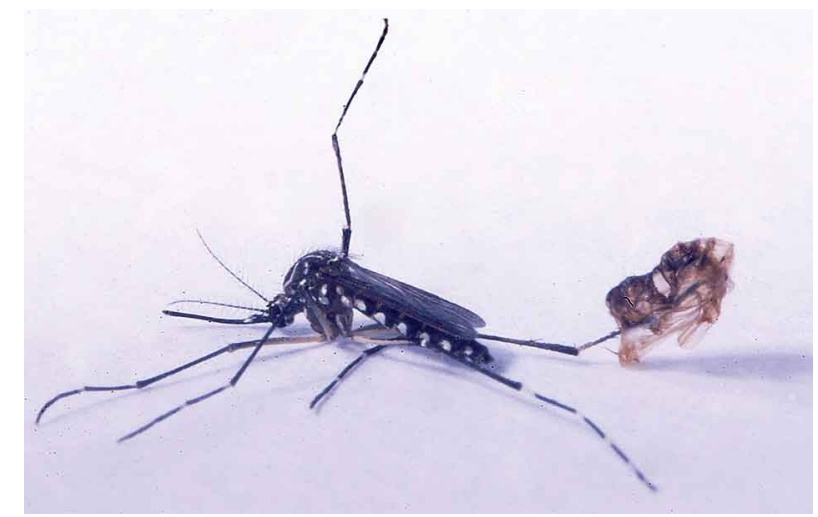

Fig. 4: Aedes aegypti female resulting from triflumuron treatment.

Triflumuron treatment interfered with the bloodfeeding ability of the surviving females; both the number of blood-fed females and the amount of ingested blood were reduced in the triflumuron-treated individuals. The same result was reported by Vasuki (1999) with other Culicidae species after treatment with the CSI hexaflumuron. In contrast to this author, we observed mortality after egglaying in a high proportion of females, probably due to their greater weakness.

Since the triflumuron-treated females ingested less blood, it was expected that the oviposition of triflumuronsurviving females would be reduced. This was effectively the case: these females laid roughly $25 \%$ fewer eggs than the control females, which is a rate compatible with the $30 \%$ reduction in the amount of blood ingested by the triflumuron-exposed group. A reduction in the oviposition of CSI-treated females of different insect orders has already been reported (Miura et al. 1976, Wilson \& Cryan 1997, Sáenz-de-Cabezón et al. 2006). However, Arias and Mulla (1975) did not detect any significant decrease in the amount of eggs laid by Culex tarsalis females treated with diflubenzuron. The number of eggs laid by methoprenesurviving Ae. aegypti females was also equivalent to the control group (Braga et al. 2005).

Eggs from a portion of the triflumuron-surviving females cracked when immersed in water (Fig. 3). Observation of the spermathecae from these females confirmed the absence of insemination. Crosses between control and triflumuron-surviving adults revealed interference with both sexes.

Our results indicate that CSIs are a viable alternative for the control of Ae. aegypti. Beyond their effectiveness against larvae (Martins et al. 2008), surviving adults exhibit a series of physiological constraints that reduce their longevity and reproductive ability. Hence, CSI survivors are expected to have a reduced vectorial capacity, whereby they are less competent in the transmission of pathogens. Furthermore, the current need for environmentally safer technologies requires the development of insecticides with more selective target sites and with a reduced risk level to non-target organisms. It has already been verified that some CSI compounds 
have little or no effect on mammals, birds and aquatic invertebrates (Mian \& Mulla 1982). Ecotoxicity studies with triflumuron are required before it can be utilised against insect vectors of medical importance.

\section{ACKNOWLEDGEMENTS}

To Diego de Lacerda Rosa, Nathalia Giglio Fontoura and Diogo Fernandes Bellinato, for technical assistance, to Genilton Vieira, for picture shown in Fig. 4, and to BayerCropscience, for the triflumuron sample.

\section{REFERENCES}

Amir OG, Peveling R 2004. Effect of triflumuron on brood development and colony survival of free-flying honeybee, Apis mellifera L. J Appl Entomol 128: 242-249.

Arias JR, Mulla MS 1975. Postemergence effects of two insect growth regulators on the mosquito Culex tarsalis (Diptera: Culicidae). J Med Entomol 12: 317-322.

Batra CP, Mittal PK, Adak T, Ansari MA 2005. Efficacy of IGR compound Starycide $480 \mathrm{SC}$ (triflumuron) against mosquito larvae in clear and polluted water. $J$ Vect Borne Dis 42: 109-116.

Braga IA, Mello CB, Peixoto AA, Valle D 2005. Evaluation of methoprene effect on Aedes aegypti (Diptera: Culicidae) development in laboratory conditions. Mem Inst Oswaldo Cruz 100: 435-440.

Clements AN 1992. The Biology of Mosquitoes, Vol. I, Chapman \& Hall, London, $509 \mathrm{pp}$

Consoli R, Lourenço-de-Oliveira R 1994. Principais mosquitos de importância sanitária no Brasil, Fiocruz, Rio de Janeiro, 225 pp.

Da-Silva JJ, Mendes J, Lomônaco C 2004. Developmental stress by diflubenzuron in Haematobia irritans (L.) (Diptera: Muscidae). Neotrop Entomol 33: 249-253.

Demark JJ, Bennet GW 1989. Efficacy of chitin synthesis inhibitors on nymphal german cockroaches (Dictyoptera: Blattelidae). $J$ Econ Entomol 82: 1633-1637.

El-Sayed FMA, Razik MA, Kandil MA 1984. Biological activity of the insect growth regulator triflumuron against Tribolium confusun (Duv.). Bull Ent Soc Egypt 14: 171-176.

Gentile C, Meireles-Filho ACA, Britto C, Lima JBP, Valle D, Peixoto AA 2006. Cloning and daily expression of the timeless gene in Aedes aegypti (Diptera: Culicidae). Insect Biochem Mol Biol 36: 878-884.

Graf JF 1993. The role of insect growth regulators in arthropod control. Parasitol Today 9: 471-474.

Gubler DJ 2002. Epidemic dengue/dengue hemorrhagic fever as a public health, social and economic problem in the 21st century. Trends Microbiol 10: 100-103.

Ishaaya I, Casida JE 1974. Dietary TH 6040 alters cuticle composition and enzyme activity of house fly larval cuticle. Pestic Biochem Physiol 4: 484-490.

Martins AJ, Belinato TA, Lima JB, Valle D 2008. Chitin synthesis inhibitor effect on Aedes aegypti populations susceptible and resistant to organophosphate temephos. Pest Manag Sci 64: 676-680.

Mello CB, Mendonça-Lopes D, Feder D, Uzeda CD, Carneiro RM, Rocha MA, Gonzales MS 2008. Laboratory evaluation of the effects of triflumuron on the development of Rhodnius prolixus nymph. Mem Inst Oswaldo Cruz 103: 839-842.

Mian LS, Mulla MS 1982. Biological and environmental dynamics of insect growth regulators (IGRs) as used against Diptera of public health importance. Residue Rev 84: 27-112.

Miura T, Schaefer CH, Takahashi RM, Mulligan FS 1976. Effects of the insect growth inhibitor, Dimilin, on hatching of mosquito eggs. J Econ Entomol 69: 655-658.

Mondal KAMSH, Parween S 2000. Insect growth regulators and their potential in the management of stored-product insect pests. Int Pest Manag Rev 5: 255-295.

Mulla MS, Thavara U, Tawatsin A, Chompoosri J, Zaim M, Su T 2003. Laboratory and field evaluation of novaluron, a new acylurea insect growth regulator, against Aedes aegypti (Diptera: Culicidae). J Vec Ecol 28: 241-254.

Post LC, de Yong BJ, Vincent WR 1974. 1-(2,6-disubstituted benzoyl)3-phenylurea insecticides: inhibitors of chitin synthesis. Pestic Biochem Physiol 4: 473-483.

Rehimi N, Soltani N 1999. Laboratory evaluation of Alsystin, a chitin synthesis inhibitor, against Culex pipiens pipiens L. (Dip.: Culicidae): effects on development and cuticle secretion. $J$ Appl Entomol 123: 437-441.

Reynolds SE 1987. The cuticle, growth and moulting in insects: the essential background to the action of acylurea insecticides. Pest Sci 20: 131-146.

Sáenz-de-Cabezón FJ, Pérez-Moreno I, Zalom FG, Marco V 2006. Effects of lufenuron on Lobesia botrana (Lepidoptera: Tortricidae) egg, larval and adult stages. J Econ Entomol 99: 427-431.

Soltani N, Delbecque JP, Delachambre J 1983. Penetration and insecticidal activity of diflubenzuron in Tenebrio molitor pupae. Pest Sci 14: 625-632.

Su T, Mulla MS, Zaim M 2003. Laboratory and field evaluations of novaluron, a new insect growth regulator (IGR), against Culex mosquitoes. J Am Mosq Control Assoc 19: 408-418.

Tunaz H, Uygun N 2004. Insect growth regulators for insect pest control. Turk J Agr Forestry 28: 377-387.

Valencia MDP, Miller LH, Mazur P 1996. Permeability of intact and dechorionated eggs of the Anopheles mosquito to water vapor and liquid water: A comparison with Drosophila. Cryobiol 33: 142-148.

Vasuki V 1992. Adult longevity of certain mosquito species after larval and pupal exposure to sublethal concentration of an insect growth regulator hexaflumuron. Southeast Asian J Trop Med Public Health 23: 121-124.

Vasuki V 1999. Influence of IGR treatment on oviposition of three species of vector mosquitoes at sublethal concentrations. Southeast Asian J Trop Med Public Health 30: 200-203.

Vasuki V, Rajavel R 1992. Influence of short time exposure to an insect growth regulator, hexaflumuron, on mortality and adult emergence of vector mosquitoes. Mem Inst Oswaldo Cruz 87: 275-283.

Wilson TG, Cryan JR 1997. Lufenuron, a chitin-synthesis inhibitor, interrupts development of Drosophila melanogaster. J Exp Zool 278: $37-44$.

Zaim M, Guillet P 2002. Alternative insecticides: an urgent need. Trends Parasitol 18: 161-163. 